\title{
SEU Analysis of Complex Circuits NASA Implemented in Actel RTAX-S FPGA Devices
}

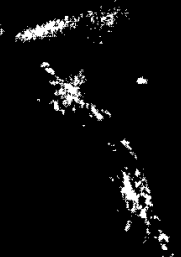

Melanie Berg, MEI Technologies/NASA GSFC

M. Friendlich, C. Perez. H. Kim, C. Seidlick. T. Irwin: MEI Technologies/NASA GSFC

K. LaBel: NASA GSFC

Place, Route, and Gate Utilization are Stored in the FPGA Configuration

Configuration Defines:

Functionality (logic cluster)

Connectivity (routes)

Placement

Configuration Switch Types:

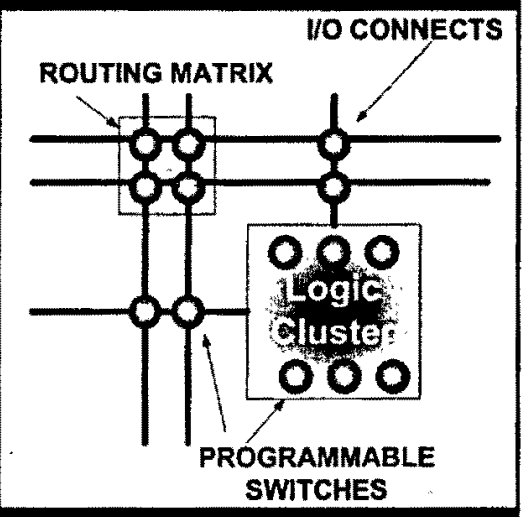

Antifuse: One time Programmable (OTP)

SRAM: Reprogrammable (RP)

Flash: Reprogrammable (RP)

To be presented by Melanie Berg at the 11th European Conference on Radiation and its effects on components and systems (RADECS) conference 9/20 to 9/24/2010, Langenfeld, Austria, and on http://radhome.gsfc.nasa.gov and http://nepp.nasa.gov/. 


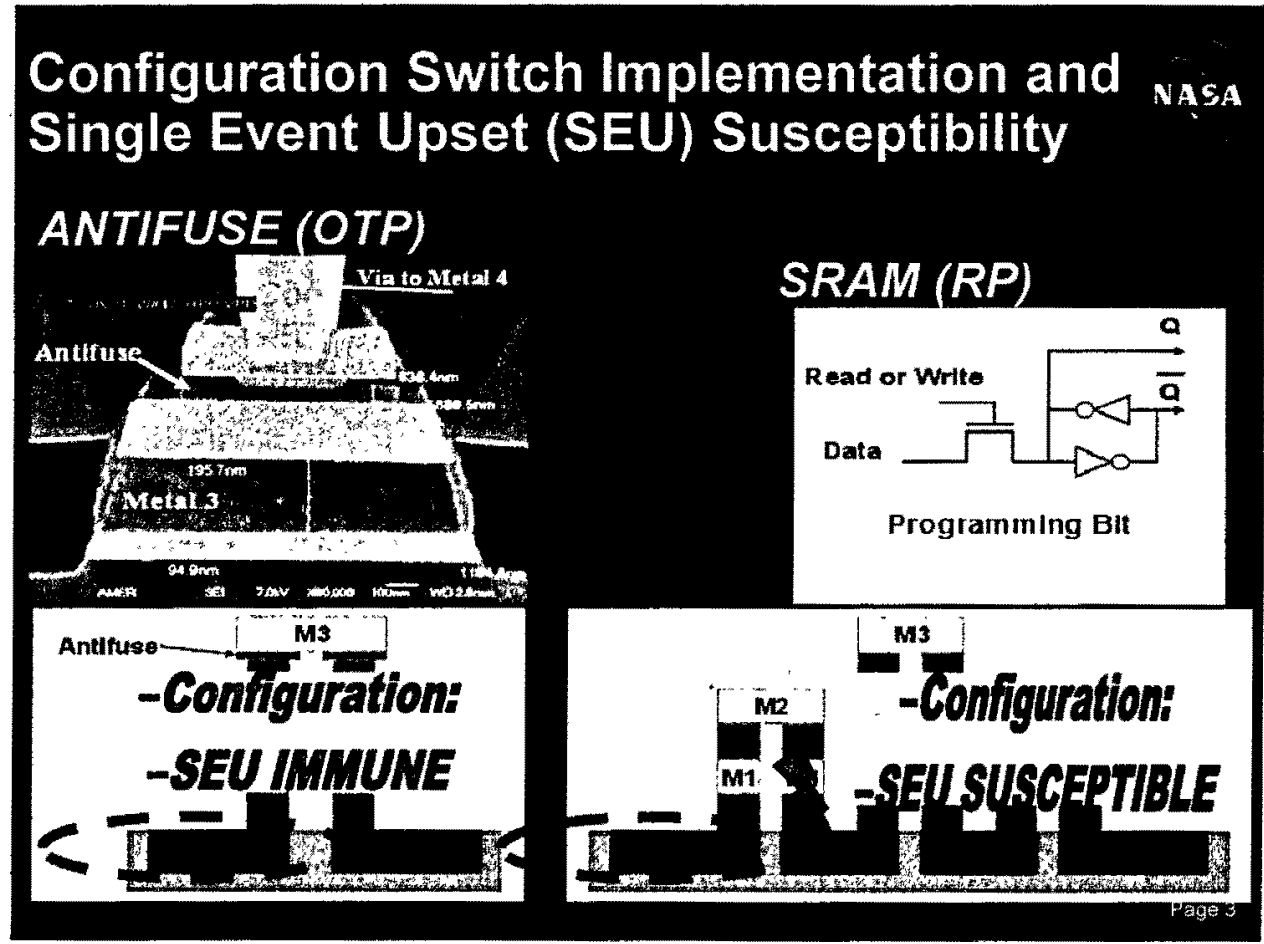

\section{Actel RTAXs -Logic Building Block} SEU and SET Susceptibility

RTAXs User Logic Blocks and Resources

- Flip-Flops (DFFs) (R-Cells)

- Combinatorial Logic (C-Cells)

- Global Routes (clocks and resets)

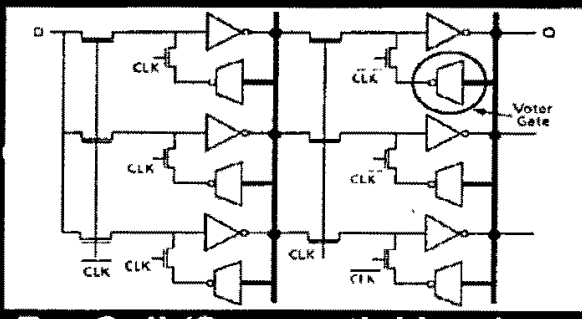

$R-$ Cell (Sequential Logic Block)

SEU:Not Frequency dependent

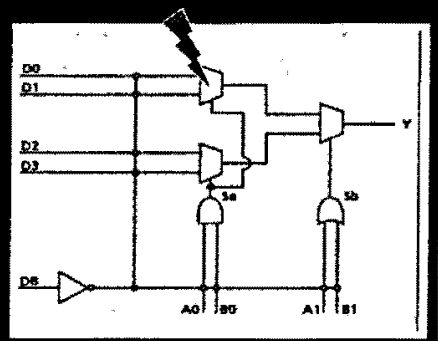

c-Cell (Combinatorial Logic Block)

SET:

Frequency dependent

To be presented by Melanie Berg at the 11th European Conference on Radiation and its effects on components and systems (RADECS) conference 9/20 to 9/24/2010, Langenfeld, Austria, and on http://radhome.gsfc.nasa.gov and http://nepp.nasa.gov/. 


\section{Actel RTAXs Susceptibility and Mitigation NasA}

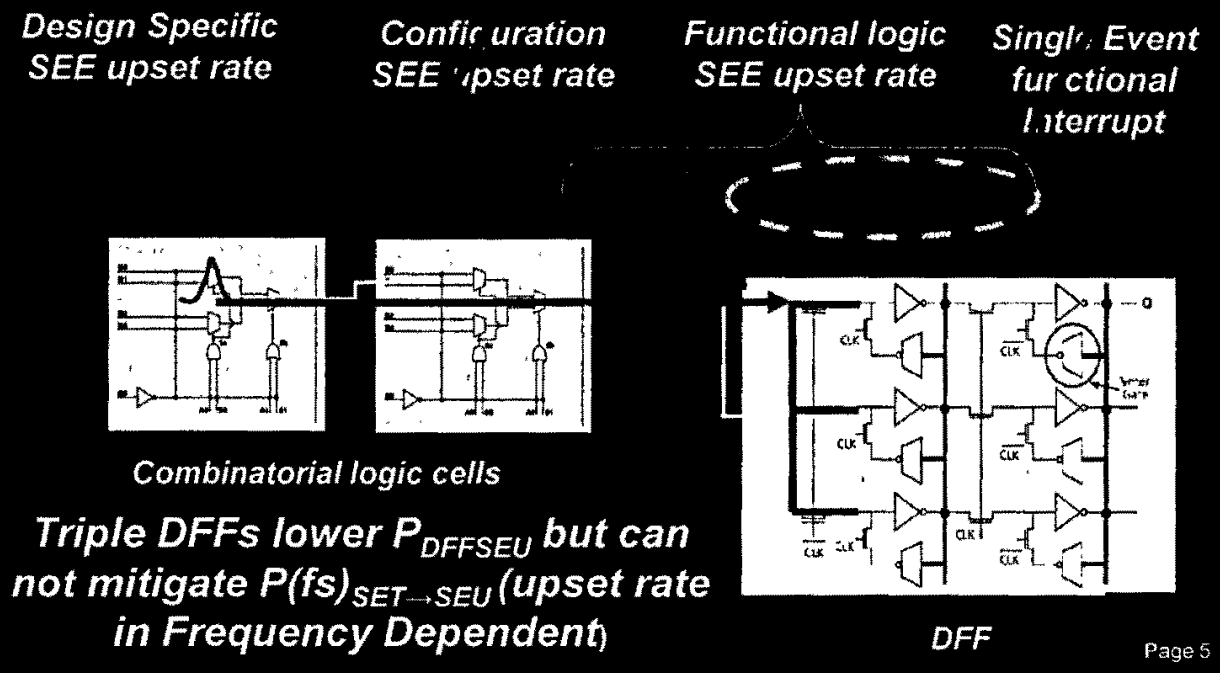

\section{FPGA Design Under Test Development

Repeatability ... increase statistics

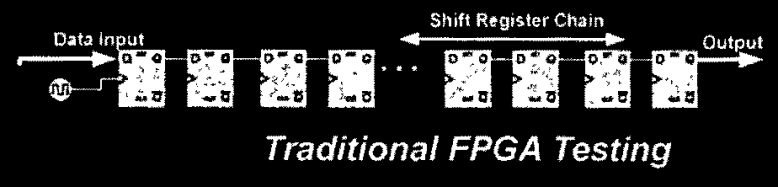

Stress and expose building blocks

Divide and conquer... Determine separate error cross sections that correspond to specific:

- Frequencies

- Designs and Building blocks (when applicable)

Fault Isolation... Create designs that will facilitate error differentiation

To be presented by Melanie Berg at the 11th European Conference on Radiation and its effects on components and systems (RADECS) conference $9 / 20$ to 9/24/2010, Langenfeld, Austria, and on http://radhome.gsfc.nasa.gov and http://nepp.nasa.gov/. 

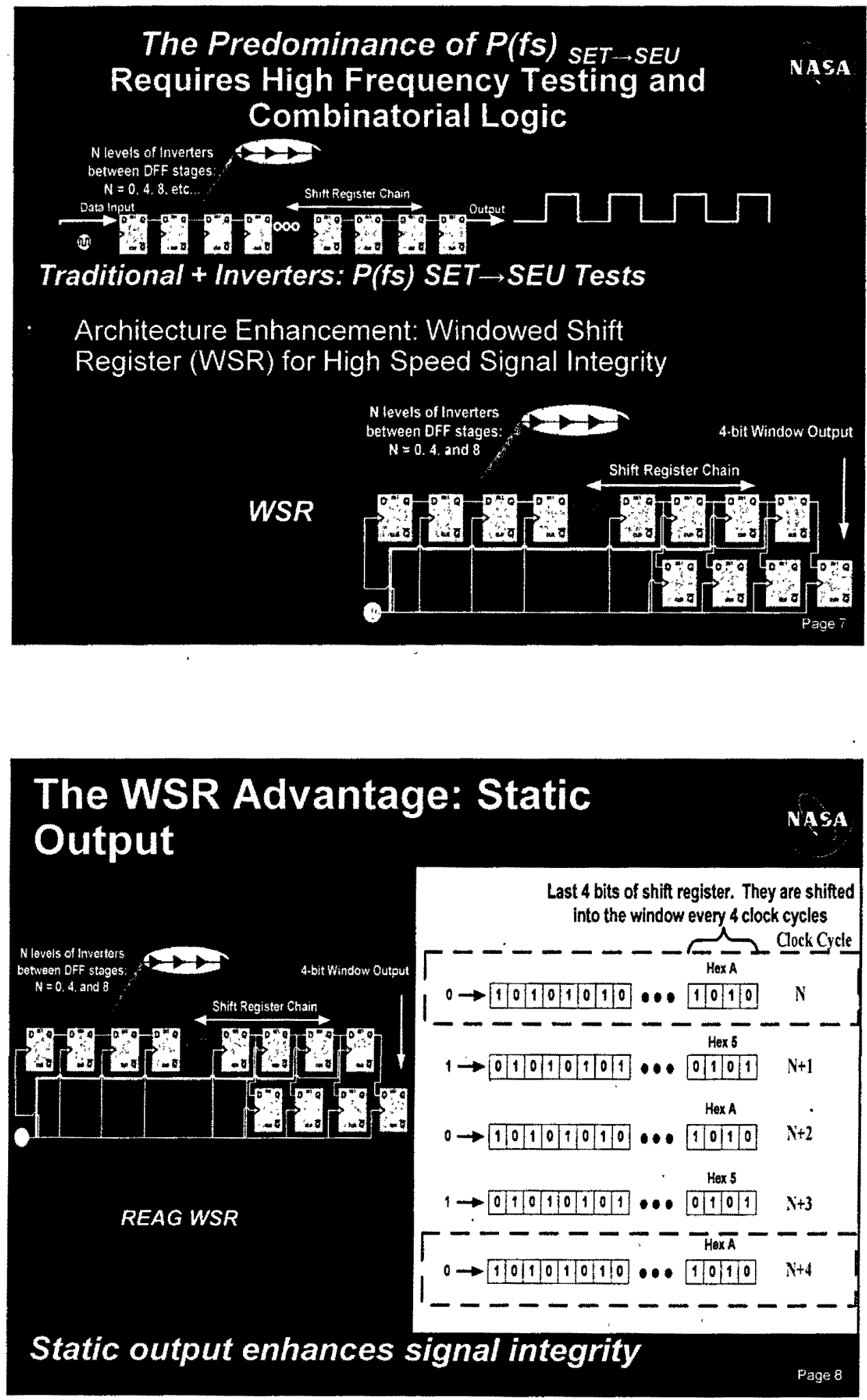

To be presented by Melanie Berg at the 11th European Conference on Radiation and its effects on components and systems (RADECS) conference 9/20 to 9/24/2010, Langenfeld, Austria, and on http://radhome.gsfc.nasa.gov and http://nepp.nasa.gov/. 


\section{Highlights/Accomplishments:}

REAG WSR SEE Results

Error rates are significantly dependent on Threshold LET(LET th )

Choice of design impacted $L_{E T}$ th ... number of C.Cells between DFFs

Choice of data pattern and frequency of operation impacted LET $_{\text {th }}$ (>2 orders of magnitude)

\begin{tabular}{|l|l|l|}
\hline & Low Frequency & Increased Frequency \\
\hline LET $_{\text {th }} \mathrm{MeV}^{*} \mathrm{~cm}^{2} / \mathrm{mg}$ & $\mathrm{LET}_{\mathrm{th}}>37$ & $8<\mathrm{LET}_{\text {th }}<30$ \\
\hline Bit Error Rate (errors/bit-day) & $\mathrm{dE}_{\mathrm{bit}} / \mathrm{dt} \approx 1 \times 10^{-10}$ & $1 \times 10^{-10<} \mathrm{dE}_{\mathrm{bit}} / \mathrm{dt}<5 \times 10^{-8}$ \\
\hline
\end{tabular}

If Frequency or data pattern were not varied during testing, then an incorrect LET th $_{\text {th }}$ and $\mathrm{dE}_{\text {bit }} / \mathrm{dt}$ would had been calculated

\section{Considerations when Developing a} Complex Design under Test Architecture

Are inverters efficient test structures?

Want to investigate something more realistic than a shift register

- It should have the characteristics of a complex design with:

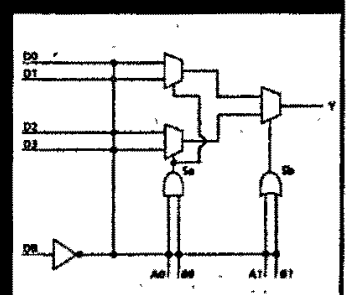

- fan-out and fan-in $>1$

- contains a mixture of sequential and combinatorial logic

- The circuit should be replicated to increase statistics.

- Its state space can be traversed within relatively short time periods such that all states are equally likely to be subject to particle strikes during radiation testing

To be presented by Melanie Berg at the 11th European Conference on Radiation and its effects on components and systems (RADECS) conference 9/20 to 9/24/2010, Langenfeld, Austria, and on http://radhome.gsfc.nasa.gov and http://nepp.nasa.gov/. 


\section{Counters Meet Requirements}

Has characteristics of a complex design with:

- fan-out and fan-in $>1$

- contains a mixture of sequential and combinatorial logic.

Variety of data pattern frequencies $\left(f_{d}\right)$

State space

Traversal $=2^{\mathrm{N}} / f_{s}$
Simplified Schematic - not actual N-bit counter with no carry logic

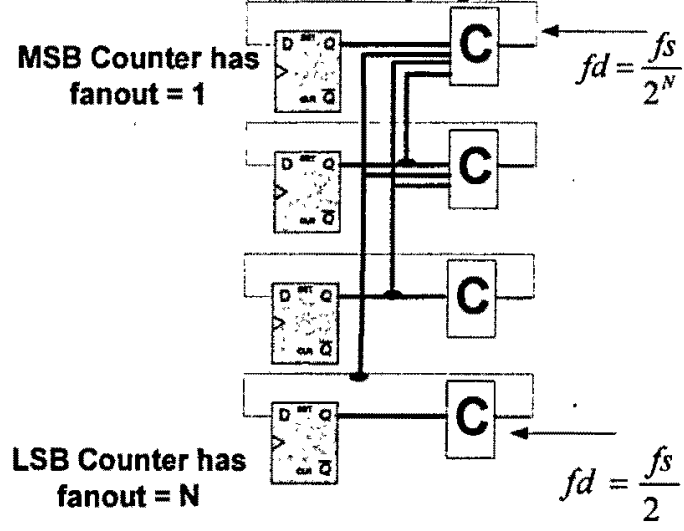

fd: data pattern frequency fs = system frequency

\section{Highlights/Accomplishments:}

Texas A\&M Heavy Ion Results Counters versus WSRs

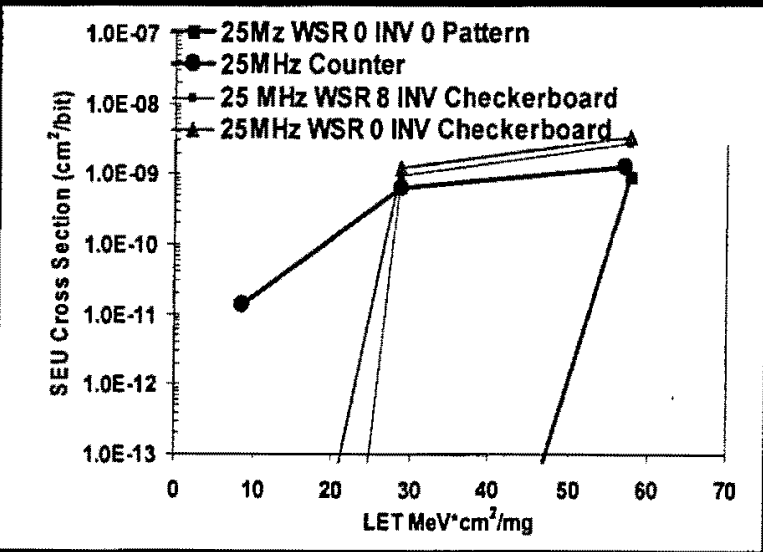

$25 M H z$ Counter LETth is lower than $25 M H z$ WSR but similar to $100 \mathrm{MHz} C B$ WSRs (also has a $L E T_{\text {th }}=$ 8. $6 \mathrm{MeV}^{*} \mathrm{~cm}^{2} / \mathrm{mg}$ )

To be presented by Melanie Berg at the 11th European Conference on Radiation and its effects on components and systems (RADECS) conference 9/20 to 9/24/2010, Langenfeld, Austria, and on http://radhome.gsfc.nasa.gov and http://nepp.nasa.gov/. 


\section{Summary}

Because configuration, global routes, and configuration is hardened in the RTAXs device, $P_{\text {SET } \rightarrow \text { SEU }}$ becomes the most significant source of upsets in radiation environments

The ratio of combinatorial logic delay to clock frequency within a data path will drive $P_{S E T \rightarrow S E U}$. Hence, Choice of Architecture and frequency can significantly affect SEE cross section and bit error rate RTAXs characterization

- Counter Arrays were driven near their top speed while containing several combinatorial logic levels. Subsequently they produced the lowest LETth $\rightarrow$ Highest bit error rate versus the shift register designs

\section{Summary: REAG Evolution of FPGA Designs Under Test}

Traditional Shift Register Testing with addition of Combinatorial logic

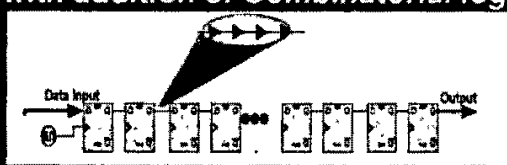

.
WSRs: High Speed Signal Integrity

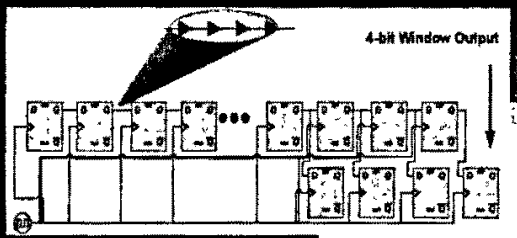

All Designs are used for all FPGA dynamic tests
Counter Arrays: More Realistic testing... not meant to replace WSRs - just an enhancement

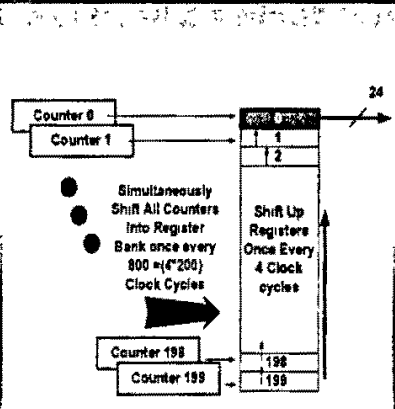

To be presented by Melanie Berg at the 11th European Conference on Radiation and its effects on components and systems (RADECS) conference 9/20 to 9/24/2010, Langenfeld, Austria, and on http://radhome.gsfc.nasa.gov and http://nepp.nasa.gov/. 